\title{
REVIEW \\ Changes of Leptin concentration in plasma in patients with spinal cord injury: A Meta-analysis
}

\author{
S Latifi ${ }^{1}$, D Koushki ${ }^{2}$, A Norouzi Javidan ${ }^{1}$, M Matin $^{1}$ and H Sabour ${ }^{1}$
}

Objective: The aim of this study was to investigate changes of leptin concentration in plasma in patients with spinal cord injury to come to a single concept by using a Meta-analysis.

Setting: Systematic Review.

Methods: Searching relevant articles was performed in Ovid data base, Medline (PubMed) EMBASE, Google Scholar, Cochrane and Scopus up to February 2013. Five articles were selected using two independent reviewers. Analysis were performed using SPSS version 18 and Comparative Meta-analysis software version 2.0.

Results: The combined analysis with confidence interval of $95 \%$ using comprehensive meta-analysis showed significant higher leptin levels in patients with spinal cord injury in comparison with able bodies $(P<0.0001)$. The effect of spinal lesion level on plasma leptin concentration was also statistically significant $(P<0.0001)$. Body mass index was positively related to plasma leptin concentration in both groups $(P<0.0001)$.

Conclusion: This Meta analysis approves increased level of leptin in spinal cord injured patients which can be due to fat distribution changes and sympathetic dysfunction in these patients. Our results also showed that patients with higher spinal lesion level have higher plasma leptin concentration.

Spinal Cord (2013) 51, 728-731; doi:10.1038/sc.2013.82; published online 3 September 2013

Keywords: leptin; spinal cord injury; meta-analysis

\section{INTRODUCTION}

Leptin is a 16 deka-protein hormone that plays a key role in the balance between energy expenditure and consumption. Leptin is one of the adipo-derived hormones ${ }^{1}$ that can be varied by body composition alternation in various pathological conditions. In fact lipoatrophy or other changes of body compositions that occur in known population (ie, HIV-positive ${ }^{2}$ and spinal cord injured ${ }^{3,4}$ ) may influence on leptin level and function. Leptin may also play a part in developing insulin resistance, ${ }^{5}$ glucose intolerance ${ }^{6}$ and increased cardiovascular diseases risks ${ }^{7,8}$ so understanding the alterations of its plasma concentration after SCI may lighten new points in this field. In patients with spinal cord injury (SCI) the elevated level of leptin in serum has been previously reported ${ }^{3,4,9-11}$ but still the need of a combined analysis of changes in leptin concentration in spinal cord injured patients thorough systematic reviews and Meta analysis were felt. Although the investigations on leptin effects and changes after spinal cord injury are extremely limited, a constant agreement on elevated level of leptin in this group of patients is noticeable. However some controversy still exists especially about the effect of injury level and body mass index (BMI) on leptin level. The purpose of this study was to combine the results of published articles about leptin level in patients with spinal cord injury and to report a single expression by interpreting these data using a Meta analysis.

\section{MATERIALS AND METHODS}

No other individuals or organizations beyond the listed authors and mentioned affiliations contributed in analysis and writing the manuscript.
Authors are the only responsible individuals in declaring accuracy of article content through the process of drafting, analyzing and editing the content of this study.

The searching process of relevant articles was performed by using key words of 'leptin' and 'spinal cord injury' in Medline (Pubmed), EMBASE, Google Scholar, Ovid, Cochrane and Scopus. The references of retrieved articles were also scanned to seek possible relevant articles which were not detected by standard searching methods. All potential relevant articles published up to February 2013 were reviewed. All searches were conducted by two different reviewers separately. Although no language limitation was enforced, no relevant article was found in any languages other than English.

By considering this fact that investigations on changes of leptin level after spinal cord injury on human models were so limited we tried not to exert strict inclusion and exclusion criteria. However our inclusion criteria were: 1. human study population, 2. Existence of a control group for a proper comparison. 3 . Adequate and suitable measurement of leptin concentration. 4. Post injury duration of at least one year. Exclusion criteria included animal models investigations, external administration of leptin as an intervention and studies with lack of comparative data. All of the selected studies were observational comparative with a control group with no intervention (case- control studies).

Final study selection was also performed by two independent reviewers. General data including number of cases, mean age, weight and BMI was retrieved as well as post injury duration and lab data. Only one of the selected articles evaluated leptin level on 24, 36 and 48 weeks after injury in patients with post injury duration mean of 3 months $^{3}$ while other articles reported these values years after injury. Due to assimilate retrieved data, only values of 48 weeks ( 1 year) after injury entered into analysis.

Categorical data were described as percentages and case numbers while continuous variables are reported as mean \pm standard deviation. Agreement

${ }^{1}$ Brain and Spinal Injury Research center (BASIR), Tehran University of Medical Sciences, Tehran, Iran and ${ }^{2}$ Iran University of Medical Sciences, Tehran, Iran Correspondence: Dr S Latifi, Researcher at Brain and Spinal Injury Research center (BASIR), Imam Hospital, Tehran University of Medical Sciences, Tehran, Iran. E-mail: Saharlatifi_med@yahoo.com

Received 21 April 2013; revised 23 June 2013; accepted 8 July 2013; published online 3 September 2013 
between reviewers was described with Cohen kappa. Statistical homogeneity was checked by chi-square test and $\mathrm{I}^{2}$ using Cochran heterogeneity statistic as $\mathrm{Q}^{12}$. We used correlation analysis with confidence interval of $95 \%$ to evaluate the difference mean and s.d. between leptin concentration in SCI patients and controlled group in included studies. Analysis was performed using SPSS version 18 and Comparative Meta-analysis software version 2.0.

\section{RESULTS}

Five articles were finally selected from the initial 15 citations as they met our inclusion criteria. All these articles performed no interventions and were merely observational. Only one of the selected study followed patients for 48 weeks. $^{3}$ All the other studies were comparative cross-sectional with existence of a matched control group. Agreement of study inclusion by Cohen Kappa was satisfactory (0.8). Included studies investigated a total number of 194 patients with spinal cord injury. Patient population included male individuals in all studies to omit the bias effect of sex hormones on leptin level. As leptin function may vary between male and female individuals, ${ }^{11,13}$ enrolling only male patients seems reasonable. Base line features of included studies along with patients characteristics are summarized in Tables 1 and 2. Common exclusion criteria in all five studies were: diabetes mellitus, eating disorders, liver disease, renal disorders, excessive alcohol intake, tobacco consumption, endocrine diseases, psychiatric disorders and intake of any medications that could affect hormone levels. Leptin concentration measurement was performed in all investigations by using commercial immuno-radiometric assay (IRMA) technique.

Almost all studies had an agreement on increased serum level of leptin in patients with SCI. A positive relationship between BMI and serum leptin concentration was previously reported by Huang and Maimoun et al. Our meta-analysis also revealed the strong effect of BMI on serum leptin concentration $(P<00001)$ which confirms the previous reports (Figure 1), however it is noticeable that BMI may not be a good representative marker for obesity in patients with $\mathrm{SCI}^{14}$ and cannot describe fat distribution in this group of patients. Wang et al. ${ }^{6}$ demonstrated also higher level of leptin in SCI patients in comparison with able bodies. They also reported a higher leptin level in tetraplegic patients than paraplegics. Although this difference was not significant, it raised the hypothesis of relationship between injury level and leptin concentration. Due to investigate this aspect Wang et al. divided injury level into two groups (above and below T6) and revealed a significant higher concentration of leptin in patient with injury level above T6. However previous studies of Maïmoun ${ }^{3}$ and Huang ${ }^{9}$ declared no significant difference in leptin level between tetraplegic and paraplegic patients. Jeon et al. ${ }^{15}$ also reported a higher serum concentration of leptin in patients with higher lesion level.

Higher concentrations of leptin level in serum were statistically significant by analysis the leptin mean \pm s.d. values in SCI and control group. The combined analysis with confidence interval of $95 \%$ using comprehensive meta-analysis showed significant higher leptin level in patients with spinal cord injury in comparison with able bodies ( $P$-value $<0.0001)$. Only one study (Maruyama et al.) was excluded during analysis due to lack of comparative data. The results are shown in Figure 2.

While Maïmoun et al. and Huang et al. demonstrated no significant difference between paraplegic and tetraplegic patients in plasma leptin level, Wang et al. showed a significant higher plasma leptin concentration in patients with higher level of spinal cord lesion. Due to higher strength of study power in Wang's study our Metaanalysis results revealed a significant association between lesion level and plasma leptin concentration (Figure 3).

\section{DISCUSSION}

Leptin is a fat-derived hormone and is affected by body fat distribution. Assessment of alterations in leptin plasma concentration after SCI may lighten new points in etiologies of increased insulin resistance, ${ }^{5}$ glucose intolerance ${ }^{6}$ and cardiovascular diseases risk ${ }^{7,8}$ which are shown to occur in spinal cord injured patients. Despite the very limited investigations in this area, the lack of systematic reviews was felt which leaded us to perform a Meta-analysis due to report a single concept in this field.

The increased level of leptin after (SCI) was reported previously. Maïmoun et al. ${ }^{3}$ reported that leptin level continuously increases when post injury duration exceeds 48 weeks. As this study was performed on patients with recent onset of spinal cord injury, no significant BMI difference existed between SCI patients and control group meaning that despite similar body composition between these two groups, higher level of leptin in spinal cord injured patients is significant. The results of this analysis show that higher level of leptin concentration in patients with spinal cord injury is statistically

Table 1 Features of included studies

\begin{tabular}{|c|c|c|c|c|c|}
\hline Study & Location/Publication Year & Design & Setting & Patient Number & Mean post injury duration \\
\hline Maïmoun et al. & France/2004 & Case-control & Single center & 7 & 3 months +1 year follow up \\
\hline Huang et al. & China/2000 & Cross-sectional comparison & Single center & 47 & 9.1 years \\
\hline Wang et al. & China/2005 & Cross-sectional comparison & Single center & 89 & 10.8 year \\
\hline Jeon et al. & Canada/2003 & Cross-sectional comparison & Single center & 7 & Not mentioned \\
\hline Maruyama et al. & Japan/2008 & Cross-sectional comparison & Single center & 44 & 26 years \\
\hline
\end{tabular}

Table 2 Baseline features of patients in included studies

\begin{tabular}{|c|c|c|c|c|c|c|}
\hline Study & Number of case & Paraplegic No. & Tetraplegic No. & Mean Age & Mean weight & Mean BMI \\
\hline Maïmoun et al. & 7 & 4 & 3 & 31.3 & $70.0 \pm 8.5$ & $22.3 \pm 2.9$ \\
\hline Huang et al. & 47 & 31 & 16 & 36.5 & & 20.9 \\
\hline Wang et al. & 89 & 59 & 30 & $39.3 \pm 1.1$ & $65.1 \pm 1.2$ & $22.3 \pm 0.4$ \\
\hline Jeon et al. & 7 & 0 & 7 & $38.3 \pm 3$ & $86.5 \pm 7$ & $26.7 \pm 1.5$ \\
\hline Maruyama et al. & 44 & 28 & 16 & $57 \pm 13$ & $64.4 \pm 13.2$ & $24.1 \pm 4.8$ \\
\hline Total & 194 & 122 & 72 & $40.48 \pm 9.73$ & $71.5 \pm 10.3$ & $23.26 \pm 2.23$ \\
\hline
\end{tabular}


significant. Most of previously published studies agree on this fact that plasma leptin correlates positively with BMI and body weight ${ }^{16-19}$ but this effect in spinal cord injured patients is mostly due to changes of fat distribution because this group of patients may have increased leptin concentration in plasma despite decreased BMI. However our Mata-analysis showed a significant strong association between

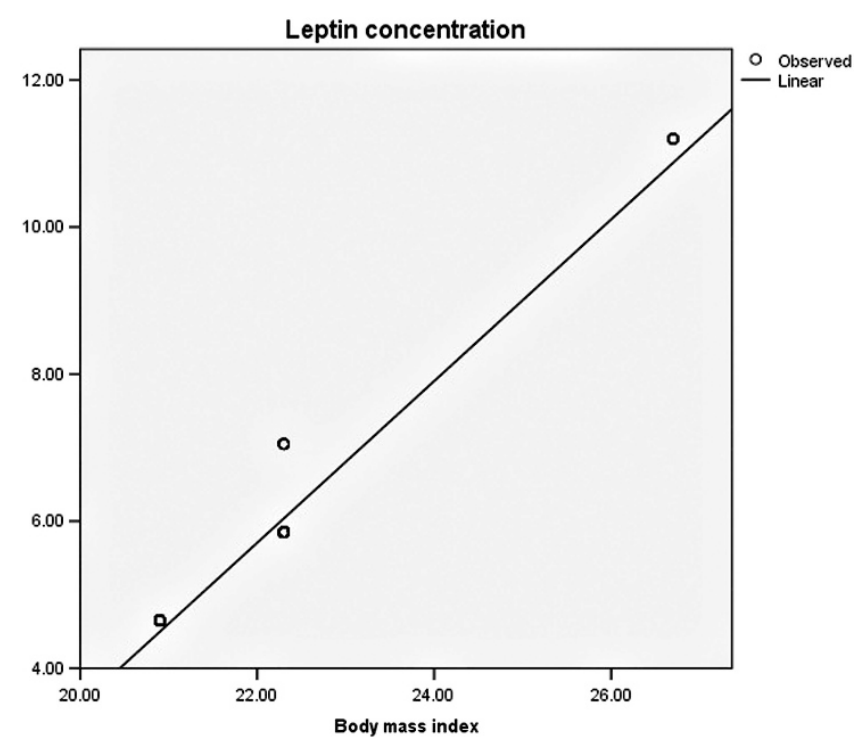

Figure 1 The linear relationship between body mass index and serum concentration of leptin in both spinal cord injured individuals and normal controls.
BMI and serum leptin concentration in both groups (Figure 1). Huang et al. ${ }^{9}$ reported that although leptin concentration is higher in patients with SCI than control group with matched BMI, body mass index was positively related with leptin level in both groups separately which is in line with Maïmoun results. Maruyama and et al. ${ }^{20}$ also showed that total lean mass is decreased in spinal cord injured patients along with increased total fat mass and visceral fat area. Although BMI seems to be a poor representative marker for obesity in SCI population, ${ }^{14}$ still the existence of a strong correlation between BMI and fat mass allows the mentioned comparison between Maiimoun and Huang results. However a positive association with plasma leptin level and BMI has been frequently reported in patients with $\mathrm{SCI}^{9,20}$ which is in line with our outcomes. By considering this fact that body composition after spinal cord injury undergoes some massive changes $^{21,22}$ including decreased total lean mass along with increased total fat mass and visceral fat area, the higher level of leptin in SCI patients was justified (Table 3). Changes of fat distribution may not be the only factor resulting in increased leptin level in spinal cord injured patients as Jeon et al. demonstrated that decreased sympathetic nerve activity that occurs in spinal cord injury leads to increased level of leptin due to higher risk of developing obesity. ${ }^{15}$ The fact that sympathetic nerve dysfunction can lead to obesity was also previously reported by Peterson et al. ${ }^{23}$

Wang et al. found a specific correlation between spinal cord lesion level and plasma leptin concentration but Maïmoun et al and Huang et al reported no significant difference between paraplegic and tetraplegic patients. Due to higher weight of Wang study (noticeable higher number of cases), the results of our Meta-analysis revealed a significant association between lesion level and plasma leptin concentrations (Figure 3). However, confirming the definite relationship

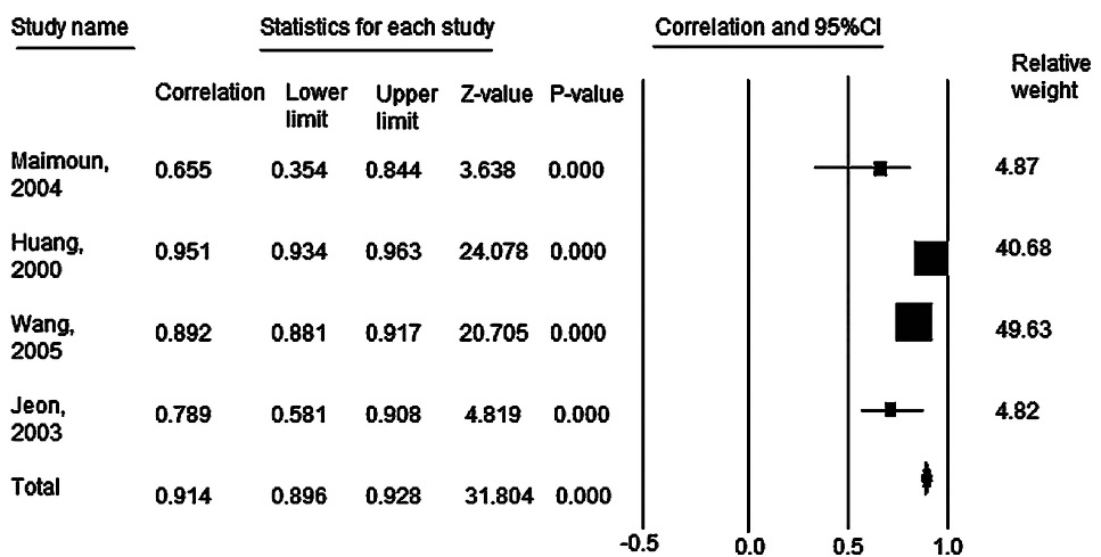

Figure 2 Meta-analysis using correlation with 95\% of confidence interval between serum leptin concentration and existence of a spinal cord lesion.

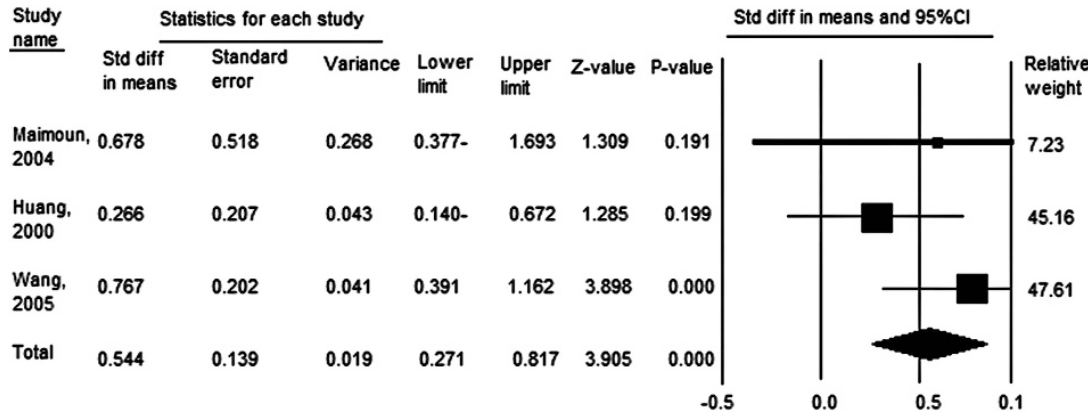

Figure 3 Meta-analysis of spinal cord lesion level effect on plasma leptin concentration. 
Table 3 Plasma leptin concentrations and main conclusions of included studies

\begin{tabular}{|c|c|c|c|}
\hline Study & Mean leptin concentration in $\mathrm{SCl}$ group & Mean leptin concentration in control group & Main conclusions \\
\hline Huang et al. & $6.23 \pm 0.66 \mathrm{ng} / \mathrm{ml}$ & $3.07 \pm 0.31$ & $\begin{array}{l}\text { Leptin level was higher in } \mathrm{SCl} \text { patients than control group } \\
\text { A linear relationship between leptin level and } \mathrm{BMI}^{\mathrm{a}}\end{array}$ \\
\hline Wang et al. & $7.0 \pm 0.5 \mathrm{ng} / \mathrm{ml}$ & $4.7 \pm 0.60$ & $\begin{array}{l}\text { Higher leptin level in } \mathrm{SCl} \text { patients than normal controls }{ }^{\mathrm{a}} \\
\text { Higher serum leptin concentration by lesion level above } \mathrm{T6}^{\mathrm{a}}\end{array}$ \\
\hline Maruyama et al. & $5.40 \mathrm{ng} / \mathrm{ml}$ & NA & $\begin{array}{l}\text { Positive relationship between leptin level and body fat composition } \\
\text { including fat mass and visceral area }{ }^{\mathrm{b}}\end{array}$ \\
\hline
\end{tabular}

Abbreviations: NA, not available; $\mathrm{SCl}$ : Spinal cord injury.

asignificance $P$-value at level of $<0.01$.

bignificance $P$-value at level of $<0.05$.

require more citations. As studies in this field are so limited, more investigations to evaluate spinal lesion level effect on leptin concentration are required.

\section{CONCLUSION}

Our meta-analysis shows that serum concentration of leptin is significantly increased in patients with SCI. although this raise is independent to BMI, body mass index have a significant positive correlation with leptin concentration in both spinal cord injured patients and normal controls. Moreover this meta-analysis insist on the effect of injury level on leptin concentration.

\section{STUDY LIMITATION}

The primary purpose of this study was to investigate alterations of leptin plasma concentration after SCI and determining factors that influence its level. Unfortunately the retrieved literatures were so limited and we could not perform proper comparison in assessing some factors that may influence on leptin concentration. This limited range of literatures has restricted our study power. However we have determined significant increased leptin plasma concentration after SCI and its relationship with injury level. Moreover further investigations including designing biomedical and experimental studies may lead to understanding biochemical pathways of these alterations.

\section{CONFLICT OF INTEREST}

The authors declare no conflict of interest.

1 Brennan AM, Mantzoros CS. Drug Insight: the role of leptin in human physiology and pathophysiology-emerging clinical applications. Nat Clin Pract Endocrinol Metab 2006; 2: 318-327.

2 Rao MN, Schambelan M, Tai VW, Abrams DI, Khatami H, Havel PJ et al. Assessing the Association between Leptin and Bone Mineral Density in HIV-Infected Men. AIDS Res Treat 2012; 2012: 103072 .

3 Maïmoun L, Puech AM, Manetta J, Badiou S, Paris F, Ohanna F et al. Circulating leptin concentrations can be used as a surrogate marker of fat mass in acute spinal cord injury patients. Metabolism 2004; 53: 989-994.

4 Bauman WA, Spungen AM, Zhong YG, Mobbs CV. Plasma leptin is directly related to body adiposity in subjects with spinal cord injury. Horm Metab Res 1996; 28 : 732-736.

5 Lee MY, Myers J, Hayes A, Madan S, Froelicher VF, Perkash I et al. C-reactive protein, metabolic syndrome, and insulin resistance in individuals with spinal cord injury. $J$ Spinal Cord Med 2005; 28: 20-25.
6 Duckworth WC, Jallepalli P, Solomon SS. Glucose intolerance in spinal cord injury. Arch Phys Med Rehabil 1983; 64: 107-110.

7 Cragg JJ, Stone JA, Krassioukov AV. Management of cardiovascular disease risk factors in individuals with chronic spinal cord injury: an evidence-based review. J Neurotrauma 2012; 29: 1999-2012.

8 Wahman K, Nash MS, Lewis JE, Seiger A, Levi R. Increased cardiovascular disease risk in Swedish persons with paraplegia: The Stockholm spinal cord injury study. J Rehabil Med 2010; 42: 489-492.

9 Huang TS, Wang YH, Chen SY. The relation of serum leptin to body mass index and to serum cortisol in men with spinal cord injury. Arch Phys Med Rehabil 2000; 81: 1582-1586.

10 Wang YH, Huang TS, Liang HW, Su TC, Chen SY, Wang TD. Fasting serum levels of adiponectin, ghrelin, and leptin in men with spinal cord injury. Arch Phys Med Rehabil 2005; 86: 1964-1968.

11 Thomas T, Burguera B, Melton LJ 3rd, Atkinson EJ, O'Fallon WM, Riggs BL et al. Role of serum leptin, insulin, and estrogen levels as potential mediators of the relationship between fat mass and bone mineral density in men versus women. Bone 2001; 29: 114-120.

12 Higgins JP, Thompson SG, Deeks JJ, Altman DG. Measuring inconsistency in metaanalyses. BMJ 2003; 327: 557-560.

13 Weiss LA, Barrett-Connor E, von Mühlen D, Clark $P$. Leptin predicts BMD and bone resorption in older women but not older men: the Rancho Bernardo Study. J Bone Miner Res 2006; 21: 758-764.

14 Buchholz AC, McGillivray CF, Pencharz PB. The use of bioelectric impedance analysis to measure fluid compartments in subjects with chronic paraplegia. Arch Phys Med Rehabil 2003; 84: 854-861.

15 Jeon JY, Steadward RD, Wheeler GD, Bell G, McCargar L, Harber V et al. Intact sympathetic nervous system is required for leptin effects on resting metabolic rate in people with spinal cord injury. J Clin Endocrinol Metab 2003; 88: 402-407.

16 Hassink SG, Sheslow DV, de Lancey E, Opentanova I, Considine RV, Caro JF. Serum leptin in children with obesity: Relationship to gender and development. Pediatrics 1996; 98: 201-203.

17 Ellis KJ, Nicolson MY. Leptin levels and body fatness in children: Effects of gender, ethnicity, and sexual development. Pediatr Res 1997; 42: 484-488.

18 Zimmet $\mathrm{P}$, Hodge A, Nicolson M, Staten M, de Lancey E, Opentanova I, Considine RV, Caro JF, Moore J et al. Serum leptin concentration, obesity, and insulin resistance in Western Samoans: Cross sectional study. Br Med J 1996; 313: 965-969.

19 Mantzoros CS, Moschos S, Avramopoulos I, Kaklamani V, Liolios A, Doulgerakis DE et al. Leptin concentrations in relation to body mass index and the tumor necrosis factor-a system in humans. J Clin Endocrinol Metab 1997; 82: 3408-3413.

20 Maruyama Y, Mizuguchi M, Yaginuma T, Kusaka M, Yoshida H, Yokoyama K et al. Serum leptin, abdominal obesity and the metabolic syndrome in individuals with chronic spinal cord injury. Spinal Cord 2008; 46: 494-499.

21 Spungen AM, Adkins RH, Stewart CA, Wang J, Pierson RN Jr, Waters RL et al. Factors influencing body composition in persons with spinal cord injury: a cross-sectional study. J Appl Physiol 2003; 95: 2398-2407.

22 Modlesky CM, Bickel CS, Slade JM, Meyer RA, Cureton KJ, Dudley GA. Assessment of skeletal muscle mass in men with spinal cord injury using dual-energy X-ray absorptiometry and magnetic resonance imaging. J Appl Physiol 2004; 96: 561-565.

23 Peterson HR, Rothschild M, Weinberg CR, Fell RD, McLeish KR, Pfeifer MA. Body fat and the activity of the autonomic nervous system. N Engl J Med 1988; 318: 1077-1083 